\title{
Detection and quantification of palm mid-fraction in a chocolate model system
}

\begin{abstract}
The tocopherol and tocotrienol compositions of the genuine cocoa butter (CB) and palm midfraction (PMF) were investigated to introduce a more reliable indicator in detecting PMF in CB. The results suggested that the $\alpha$-tocotrienol data presented could be utilised for the detection of the PMF admixture to $\mathrm{CB}$. The PMF was added to CB at different levels. HPLC was used to detect the presence of PMF admixture to $\mathrm{CB}$ using $\alpha$-tocotrienol as an indicator. The results derived from the model system indicated that increasing the PMF amount at 0 $15 \%$ to $\mathrm{CB}$ resulted in an increase in the concentration of the $\alpha$-tocotrienol significantly $(\mathrm{P}<$ $0.05)$. The addition of PMF amount more than $15 \%$ did not have any effect on the $\alpha$ tocotrienol concentration. A linear plot with a high correlation of 0.9967 was obtained with $\mathrm{SE}$ of 1.527. The high correlation obtained indicated good accuracy, reflecting a close relationship between experimental and theoretical predicted value.
\end{abstract}

Keyword: Chocolate, cocoa butter, palm mid-fraction, tocopherol, tocotrienol 